\title{
Re-thinking the relationship between food insecurity, health and social isolation
}

\author{
J.S. McKenzie and D.C. Watts \\ Rowett Institute, University of Aberdeen, Aberdeen, AB25 2ZD
}

Food insecurity is one of the starkest markers of inequality and a growing social issue in high-income countries ${ }^{(1)}$. Current research tends to focus on the detrimental impacts that food insecurity can have on the health and social networks of those who experience it ${ }^{(2)}$.

This paper uses qualitative evidence to argue that the relationship between food insecurity and individuals' perceptions of their health and social networks is more complex than much current literature suggests. It is based on in-depth interviews with fifty-five adults across Scotland who have experienced food insecurity. Participants were recruited through the Scottish Health Survey $(n=23)$ and foodbanks $(n=32)$ and the interviews took place either face-to-face or by telephone.

Focusing on emergent themes from the interview data related to the relationship between food insecurity and health and social networks, this paper demonstrates how food insecurity is experienced in different ways and exemplifies its relationships with perceived health and social networks. Themes included: health and social isolation as a cause of food insecurity; health and social isolation as a consequence of food insecurity; health and social networks as a means out of food insecurity. It argues that poor health (almost all respondents) and social isolation (about 50\% of respondents) can be both a cause and consequence of food insecurity. It will also demonstrate that there can be occasions when food insecurity is perceived to improve health (5\% of respondents); when poor health can provide a path out of food insecurity ( $5 \%$ of respondents); and that coping with food insecurity can provide access to new social networks that can also be a means to greater food security (approximately 50\% of respondents). However, it will also be demonstrated that such positive consequences of food insecurity may not be available to those who experience the most severe levels (approximately $10 \%$ of respondents).

The paper concludes that the causes and consequences of food insecurity are highly variable and that different kinds of support may be required to help those who experience it to realise food security. A greater understanding of the complexities in the lived experience of food insecurity is required if effective policies aimed at addressing this serious social inequality are to be developed.

1. Cooper N, Purcell S and Jackson R (2014) The Trussell Trust

2. Douglas F, Ejebu O, Garcia A et al. (2015) NHS Health Scotland 